\title{
Molybdenum phosphide as an efficient electrocatalyst for the hydrogen evolution
} Cite this: Energy Environ. Sci., 2014, 7, reactiont
2624

Received 25th March 2014 Accepted 28th May 2014

\author{
Peng Xiao, ${ }^{a}$ Mahasin Alam Sk, ${ }^{a}$ Larissa Thia, ${ }^{c d}$ Xiaoming Ge, ${ }^{b}$ Rern Jern Lim, ${ }^{a}$ \\ Jing-Yuan Wang, ${ }^{\mathrm{C}}$ Kok Hwa Lim ${ }^{\mathrm{a}}$ and Xin Wang*a
}

DOI: $10.1039 / c 4 e e 00957 f$

www.rsc.org/ees

Electrochemical production of hydrogen from water has been directed to the search for non-noble metal based and earth-abundant catalysts. In this work, we propose a novel cost-effective catalyst, molybdenum phosphide that exhibits high activity towards the hydrogen evolution reaction (HER) in both acid and alkaline media even in bulk form. Comparative analysis of $\mathrm{Mo}, \mathrm{Mo}_{3} \mathrm{P}$ and $\mathrm{MoP}$ as catalysts for HER clearly indicates that phosphorization can potentially modify the properties of the metal and different degrees of phosphorization lead to distinct activities and stabilities. Theoretical calculations by density functional theory also show that a simple phosphorization of molybdenum to form MoP introduces a good ' $\mathrm{H}$ delivery' system which attains nearly zero binding to $\mathrm{H}$ at a certain $\mathrm{H}$ coverage. With the combination of experimental results and theoretical calculations, this work has enlightened a new way of exploring cost-effective catalysts for HER.

\section{Introduction}

Hydrogen production through electrochemical water-splitting has been extensively pursued as it could potentially achieve sustainable fuel production and the electricity required could be obtained from renewable energy. ${ }^{1}$ Noble metals, e.g. Pt, demonstrate exceptional behavior with nearly zero overpotential in acidic media. ${ }^{2-4}$ However, their high cost and scarcity impede their widespread usage and direct our attention to earth-abundant metals or their compounds. Non-noble

${ }^{a}$ School of Chemical and Biomedical Engineering, Nanyang Technological University, 50 Nanyang Avenue, 639798, Singapore. E-mail: WangXin@ntu.edu.sg; Fax: +65 67947553

${ }^{b}$ Institute of Materials Research and Engineering (IMRE), Agency of Science, Technology, and Research (A*STAR), 3 Research Link, Singapore 117602, Singapore ${ }^{c}$ Residues and Resource Reclamation Centre, Nanyang Technological University, Singapore

${ }^{d}$ Interdisciplinary Graduate School, Nanyang Technological University, 50 Nanyang Avenue Block S2 - B3a-01, Singapore 639798, Singapore

$\dagger$ Electronic supplementary information (ESI) available. See DOI: 10.1039/c4ee00957f

\section{Broader context}

Hydrogen is currently pursued as a potential energy source and the electrochemical water splitting process has been proposed as a promising and clean means for large scale hydrogen production. Limited by the high cost and scarcity of noble metal catalysts, non-noble metal catalysts are being explored as possible alternatives, with a few successful cases to date, e.g. $\mathrm{MoS}_{2}$. However, the synthesis of these reported catalysts typically involve sophisticated methodologies in order to achieve desired nanostructures. Herein, we report a non-noble metal catalyst for the hydrogen evolution reaction (HER), molybdenum phosphide. Its synthesis can be realized via a facile two-step-sintering method. Our experimental results show that even in its bulk form, molybdenum phosphide exhibits high performance in both acidic and alkaline conditions. Furthermore, the observed performance of the bulk MoP was also comparable to that of the aforementioned nanostructured catalysts. Theoretical calculations reveal that phosphorization can potentially modify the properties of the metal and different degrees of phosphorization lead to distinct activities and stabilities.

electrocatalysts can be categorized into two categories: (1) the derivatives of organic liganded metals (e.g. Ni, Mo, Fe), such as hydrogenases or metalloenzyme, the mimics of active site of hydrogenases, ${ }^{5,6}$ molybdenum-oxo, ${ }^{5}$ and pyrene-functionalized nickel complexes; ${ }^{7}(2)$ inorganic compounds of metals. $\mathrm{MoS}_{2},{ }^{8-16}$ as a typical inorganic compound for hydrodesulfurization, ${ }^{17}$ was discovered to possess biomimetic active sites as hydrogenases. $^{\mathbf{1 8 , 1 9}}$ Selenide, ${ }^{\mathbf{2 0 , 2 1}}$ nickel molybdenum nitride, ${ }^{22}$ carbide, ${ }^{23-26}$ nickel phosphide, ${ }^{27,28}$ and first-row of transitional metal dichalcogenides ${ }^{29}$ are reported to be promising electrocatalysts towards the hydrogen evolution reaction (HER). By comparison, those cost-effective catalysts as the potential substitutes for Pt are either functionalized by organic ligands, e.g. [NiFe] hydrogenase or inorganics e.g. $\mathrm{MoS}_{2}, \mathrm{Ni}_{2} \mathrm{P}$. In a typical 'volcano plot' of hydrogen adsorption energy of common metals $(\mathrm{M}=\mathrm{Nb}, \mathrm{Mo}, \mathrm{Ni}, \mathrm{Pt}, \mathrm{Au}, \mathrm{Ag}$, etc. $),{ }^{30-32}$ it is revealed that strong metal-hydrogen (Mo, Ni, etc.) bonds could impede hydrogen release from the active sites, compared to Pt group metals, e.g. $\mathrm{Pd}$ and Pt, which excludes the non-noble metals (e.g. Ni, Mo) as suitable candidates to catalyze the hydrogen evolution reaction 
(HER). However, a subsequent study suggested that inorganic compounds of the non-noble metals can modify the metalhydrogen bond strength and achieve a Pt-resembling Gibbs free energy for hydrogen evolution in an acidic environment. The study of $\mathrm{MoS}_{2}$ and $\mathrm{Ni}_{2} \mathrm{P}$ has successfully underpinned this theoretical calculation. ${ }^{18,33}$ Inspired by this, herein, we report a molybdenum compound, MoP as a highly efficient catalyst for HER both in acidic and alkaline media. Molybdenum phosphide is synthesized though facile sintering of molybdenum and phosphorus precursors assisted by citric acid. Unlike $\mathrm{MoS}_{2}$ which shows very poor activity in bulk form, MoP still exhibits high electrocatalytic activity in bulk form. Furthermore, it was also experimentally demonstrated that going from metal Mo, $\mathrm{Mo}_{3} \mathrm{P}$ to $\mathrm{MoP}$, different degree of phosphorization results in distinct performance and different stability. Theoretically, we employed density functional theory (DFT) calculations to elucidate the underlying reasons behind the distinct performance by comparing the hydrogen chemisorption process and Gibbs free energy of $\mathrm{Mo}, \mathrm{Mo}_{3} \mathrm{P}$ and $\mathrm{MoP}$.

\section{Results and discussion}

$\mathrm{Mo}_{3} \mathrm{P}$ and MoP were synthesized at $800{ }^{\circ} \mathrm{C}$ and $650{ }^{\circ} \mathrm{C}$ respectively via a two-step sintering method (Experimental section, ESI $\dagger$ ), whilst metal Mo was obtained by reducing $\mathrm{MoO}_{3}$ in $\mathrm{H}_{2}$ at $850{ }^{\circ} \mathrm{C}$. Their corresponding XRD patterns are shown in Fig. 1. The peaks are well indexed to the standard XRD profiles and no major impurities are detected. Metal Mo, in a cubic pack (ICSD code: 643962 ) belongs to $\operatorname{Im} \overline{3} m$ (229). After the incorporation of phosphorus to form $\mathrm{Mo}_{3} \mathrm{P}$, it evolves into a tetragonal structure. Its XRD pattern confirms its $I \overline{4} 2 m$ (121) space group in Fig. 1b, with the Mo atom being either 2 or 4 -coordinated by $\mathrm{P}$ atoms. Further phosphorization leads to MoP, in an hexagonal structure $(P \overline{6} \mathrm{~m} 2)$, with Mo 6-coordinated by $\mathrm{P}$ atoms, as shown in Fig. 1c. A visualized structure change is presented in Scheme 1.

Field-emission scanning electron microscopy (FE-SEM) and transmission electron microscopy (TEM) were applied to examine its morphology. FE-SEM graphs in Fig. 2a show that the as-synthesized $\mathrm{MoP}$ and $\mathrm{Mo}_{3} \mathrm{P}$ (Fig. S3 $\dagger$ ) are in bulk form due to the calcination at high temperature. The high-resolution TEM (HR-TEM) graph exhibits a well-arrayed (001) plane of MoP

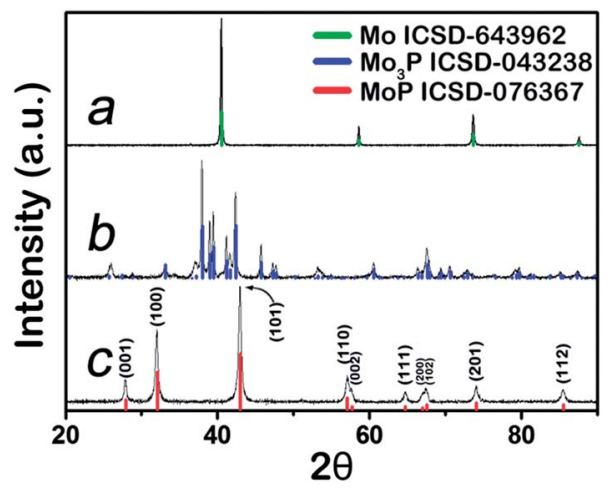

Fig. 1 XRD patterns of (a) Mo; (b) $\mathrm{Mo}_{3} \mathrm{P}$; (c) MoP.

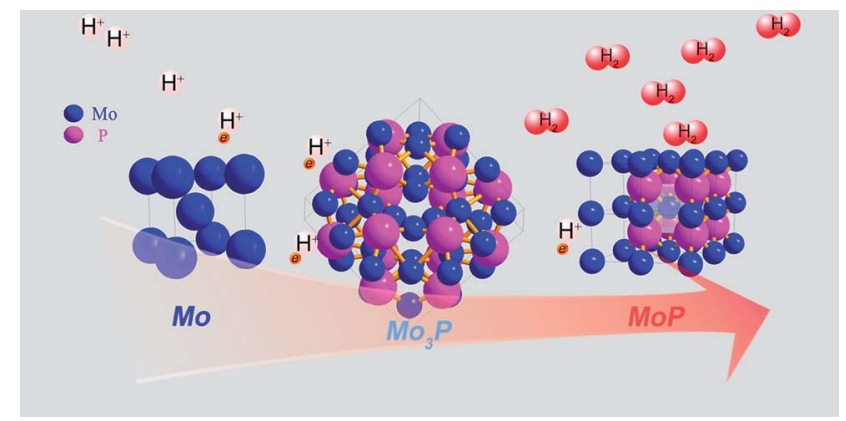

Scheme 1 Schematic graph to show the structural evolution upon phosphorization.

with a plane distance of $0.32 \mathrm{~nm}$ (Fig. 2b), which is correlated with the peak at $28^{\circ}$ in the XRD characterization. The energy dispersive X-ray spectroscopy (EDS) results confirm the compositions of $\mathrm{MoP}$ and $\mathrm{Mo}_{3} \mathrm{P}$ (Fig. S4 $\dagger$ ), which is quite consistent with the chemical formulas, $1: 1$ and $3: 1$ for MoP, $\mathrm{Mo}_{3} \mathrm{P}$ respectively. To elucidate the valence states of individual elements of MoP, X-ray photoelectron spectroscopy (XPS) experiments were conducted. Fig. $3 \mathrm{a}$ and $\mathrm{b}$ show the XPS profiles of Mo 3d and P 2p of the as-synthesized MoP. Deconvolution of the spectra indicates two doublets at $235.28 \mathrm{eV} /$ $232.08 \mathrm{eV}\left(\mathrm{Mo}^{6+} 3 \mathrm{~d}_{3 / 2} / 3 \mathrm{~d}_{5 / 2}\right)$ and $231.9 \mathrm{eV} / 228.8 \mathrm{eV}\left(\mathrm{Mo}^{4+} 3 \mathrm{~d}_{3 / 2} /\right.$ $3 \mathrm{~d}_{5 / 2}$ ) in Fig. 3a, which can be assigned to high oxidation state of Mo $\left(\mathrm{MoO}_{3}\right.$ and $\left.\mathrm{MoO}_{2}\right){ }^{34,35}$ We believe that the oxidation only happens on the surface. To validate our hypothesis, we characterize the aged MoP (Fig. S5 $\dagger$ ) by XRD, which still manifests the well-crystallized MoP and does not indicate any discernible peaks of the oxides, implying a mere surface-oxidation (less than $10 \mathrm{~nm}$ ) that could only be detected by XPS. The rest of the XPS profile depicted in green (Fig. 3a) is ascribed to MoP, represented by a $231.2 \mathrm{eV} / 228.0 \mathrm{eV}$ doublet, which agrees well with previous reports. ${ }^{36,37}$ The profile of $\mathrm{P} 2 \mathrm{p}$ also exhibits a peak with high binding energy (peak 1 in Fig. 3b) which could be attributed to $\mathrm{PO}_{4}{ }^{3-}$ or $\mathrm{P}_{2} \mathrm{O}_{5}$ (ref. 36) caused by oxidation. To identify the rest peaks for MoP, we further compared the XPS profile of the as-prepared MoP sample after testing with the aged sample. As shown in Fig. 3d, the $\mathrm{P} 2 \mathrm{p}$ profile of the aged sample does not display the low-binding-energy peaks as we observe in Fig. $3 \mathrm{~b}$. On the contrary, it manifests even stronger peaks in the lowbinding-energy region for the sample after testing (Fig. S6 $\dagger$ ). Considering the above, we assign the doublet (130.1/129.2 eV for peak 2/peak 3) to low valence of $\mathrm{P}$, MoP in this case.

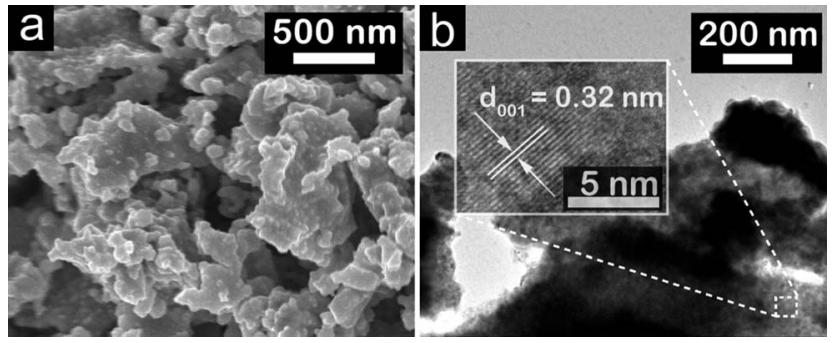

Fig. 2 Morphology of MoP (a) FE-SEM and (b) TEM graph. 

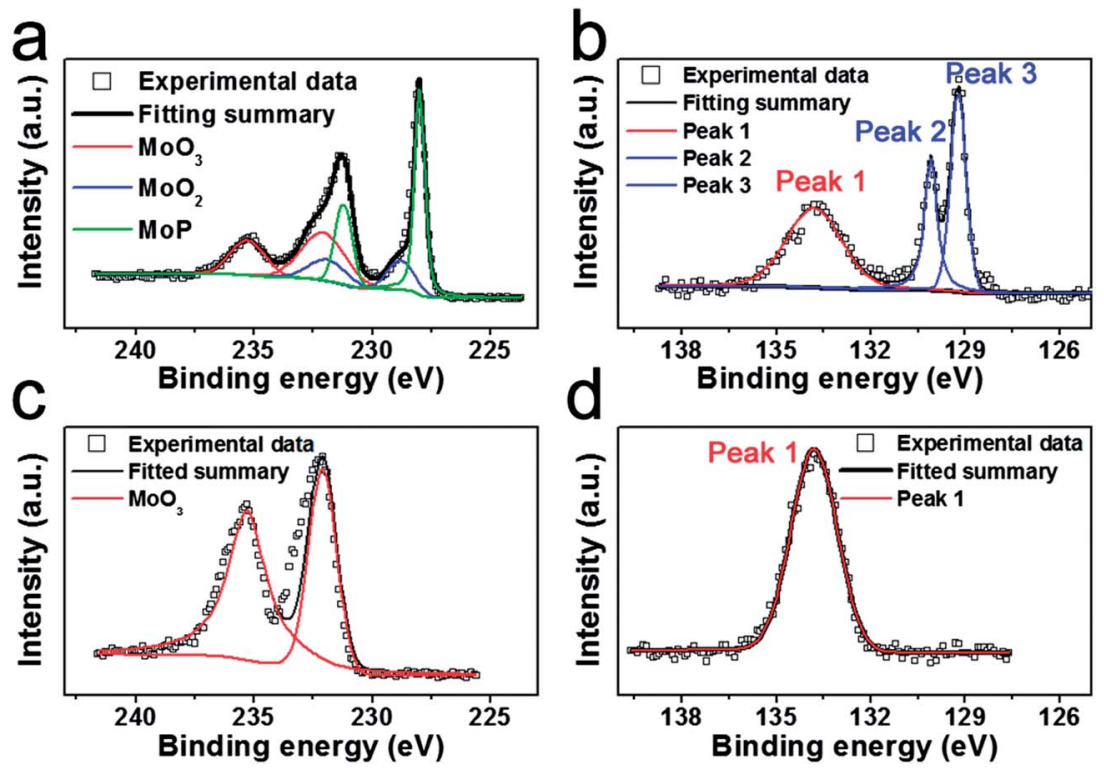

Fig. 3 X-ray photoelectron spectra (XPS) of the as-synthesized MoP (a) Mo 3d and (b) P 2p; aged MoP (c) Mo 3d and (d) P 2p.

In a three-electrode configuration, the catalytic activity towards HER was investigated in acid and alkaline media. Fig. 4 shows the performance of $\mathrm{Mo}, \mathrm{Mo}_{3} \mathrm{P}$ and $\mathrm{MoP}$ in acidic medium; $\mathrm{Pt} / \mathrm{C}$ was also tested as a comparison. The polarization curve and Tafel slope $\sim 30 \mathrm{mV} \mathrm{dec}^{-1}$ of $\mathrm{Pt} / \mathrm{C}$ presented in Fig. $4 \mathrm{a}$ and $\mathrm{b}$ are consistent with previous studies. ${ }^{38}$ After being dropcast on a glassy carbon electrode (which exhibits negligible activity towards HER), to achieve a current density of $10 \mathrm{~mA} \mathrm{~cm}{ }^{-2}$, metal Mo requires a potential of up to $0.5 \mathrm{~V} v s$.
RHE with an onset potential of $\sim 0.3 \mathrm{~V}$ vs. RHE, and $\mathrm{Mo}_{3} \mathrm{P}$ is found to perform similarly as shown by the green line, indicating that metal Mo itself is not a good catalyst for HER and its phosphide $\mathrm{Mo}_{3} \mathrm{P}$ is not suitable for HER either. In contrast, $\mathrm{MoP}$ shows a rather high performance in $0.5 \mathrm{M} \mathrm{H}_{2} \mathrm{SO}_{4}$. The onset of the hydrogen evolution reaction can be observed at around 50 $\mathrm{mV}$ vs. RHE and the current density reaches $30 \mathrm{~mA} \mathrm{~cm}{ }^{-2}$ at a potential of $0.18 \mathrm{~V}$ vs. RHE. To clarify these differences in performance, from the perspective of an elementary step, i.e.
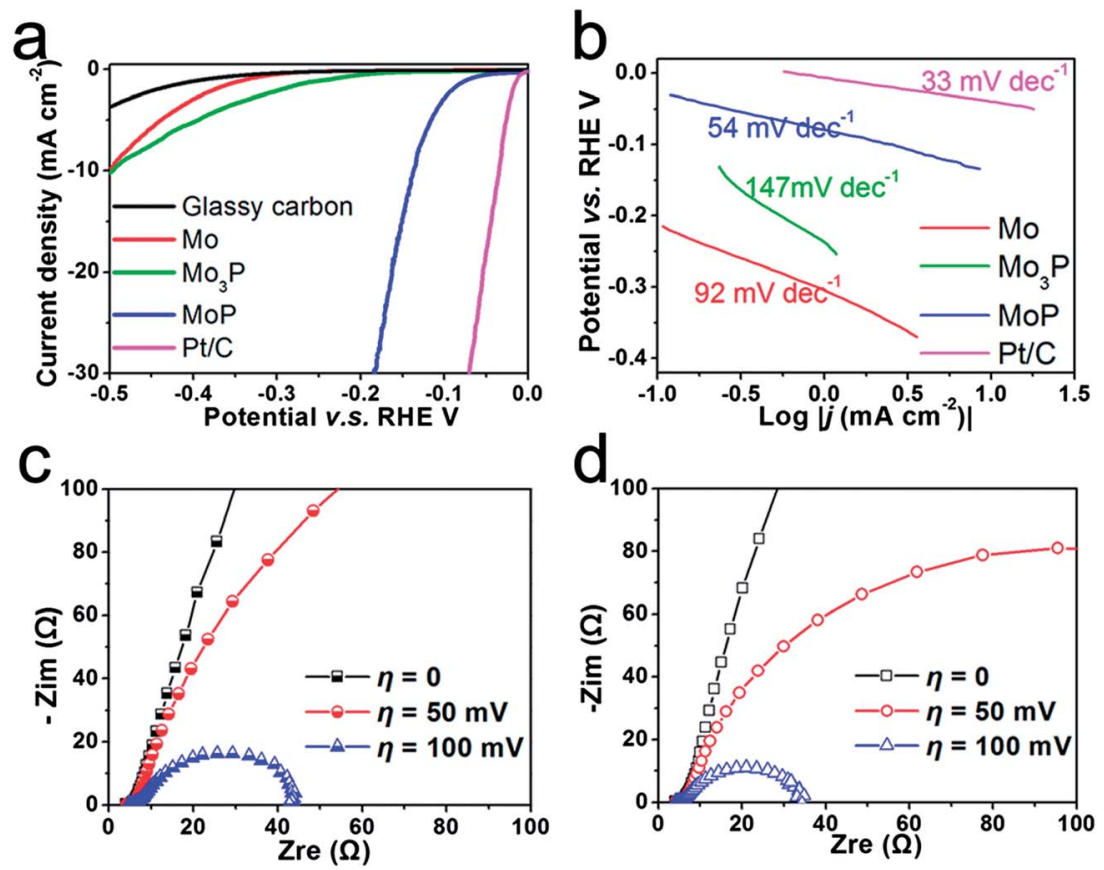

Fig. 4 (a) Polarization curves of $\mathrm{Mo}, \mathrm{Mo}_{3} \mathrm{P}$ and $\mathrm{MoP}$ in $0.5 \mathrm{M} \mathrm{H}_{2} \mathrm{SO}_{4}$, scan rate: $2 \mathrm{mV} \mathrm{s}^{-1}$; (b) Tafel plots in $0.5 \mathrm{M} \mathrm{H}_{2} \mathrm{SO}_{4}$; electrochemical impedance spectra of MoP: (c) in $0.5 \mathrm{M} \mathrm{H}_{2} \mathrm{SO}_{4}$ and (d) in $1 \mathrm{M} \mathrm{KOH}$. 
hydrogen chemisorption, we use Gibbs free energy $\left(\Delta G_{\mathrm{H}}{ }^{\circ}\right)$ as the descriptor to assess the binding strength of catalysts to $\mathrm{H}$ (e.g. Mo and MoP) and evaluate the catalysts (detailed DFT calculations can be found in the ESI $\dagger$ ). Metal Mo exhibits strong binding to $\mathrm{H}$, indicated by the highly negative binding energy $\left(\Delta E_{\mathrm{H}}\right)$ in Table $\mathrm{S} 1 \dagger$ when $\mathrm{H}$ coverage reaches $1 / 4$ of a monolayer (ML, one hydrogen adsorbed on $2 \times 2$ slab). This agrees well with the previous report. ${ }^{18}$ Calculation of the Mo terminated surface on (001)-MoP also shows it has similar or even stronger binding to $\mathrm{H}$ which excludes Mo as the active site. Nevertheless, the investigation of $\mathrm{P}$ sites implies that $\mathrm{P}$ has played a crucial role by acting as a 'hydrogen deliverer'. As shown in Table $\mathrm{S} 2, \dagger \Delta G_{\mathrm{H}}{ }^{\circ}$ changes from $-0.36 \mathrm{eV}$ to $0.54 \mathrm{eV}$ when $\mathrm{H}$ coverage increases from $1 / 4 \mathrm{ML}$ to full coverage, indicating that $\mathrm{P}$ could bond hydrogen at low coverage whilst desorb $\mathrm{H}$ at high coverage. This enables $\mathrm{P}$ to behave like a 'hydrogen deliverer', resembling the S-edges in $\mathrm{MoS}_{2} \cdot{ }^{39}$ Interestingly, when we applied the same method to calculate the binding energy of $\mathrm{H}$ on (001) of $\mathrm{Mo}_{3} \mathrm{P}$, where a four-P-site slab is chosen, the binding energy $\Delta E_{\mathrm{H}}$ becomes positive (Table $\mathrm{S} 3 \dagger$ ) with the initial two $\mathrm{H}$ adsorbed, indicating unfavorable binding of $\mathrm{H}$ on the $\mathrm{P}$ sites of $\mathrm{Mo}_{3} \mathrm{P}$. This is in accordance with the experimental results.

After electrochemical testing, the catalyst composition was again examined by XPS. Compared to the as-synthesized MoP, the XPS profile of the post-test catalysts displays a similar profile to Mo 3d and P 2p as shown in Fig. S6† except for the decreased content of $\mathrm{MoO}_{3}$ and $\mathrm{MoO}_{2}$. A previous study of $\mathrm{Mo}_{2} \mathrm{C}$ by $\mathrm{Hu}$ et al. ${ }^{23}$ has proved that $\mathrm{MoO}_{3}$ and $\mathrm{MoO}_{2}$ are not efficient catalysts, which further suggests the high performance could be attributed to MoP.

Tafel plots of potential $V-\log \mid j$ (current density) $\mid$ could be interpreted by the Volmer-Tafel or Heyrovsky mechanism in the classical two-electron-reaction model for cathodic HER. ${ }^{24}$ The Tafel slope of $92 \mathrm{mV} \mathrm{dec}^{-1}$ (Fig. 4b) for metal Mo suggests a typical Volmer-Tafel route with the Volmer step as the ratedetermining step. After phosphorization, the plot exhibits slopes of $147 \mathrm{mV} \mathrm{dec}{ }^{-1}$ and $54 \mathrm{mV} \mathrm{dec}{ }^{-1}$ for $\mathrm{Mo}_{3} \mathrm{P}$ and $\mathrm{MoP}$ respectively. Here, the Tafel slope of $54 \mathrm{mV} \mathrm{dec}^{-1}$ implies that hydrogen evolution on MoP undergoes a Heyrovsky mechanism which is still different from the route noble metals, e.g. Pt, are subjected to. Based on the Langmuir Isotherm model, ${ }^{\mathbf{4 0}}$ a fast discharging reaction (Volmer reaction) rate under low coverage $(<0.1)$ of adsorbed hydrogen $\left(\mathrm{H}_{\mathrm{ad}}\right)$ leads to $\sim 30 \mathrm{mV} \mathrm{dec}{ }^{-1}$ and the Tafel slope of $54 \mathrm{mV} \mathrm{dec}{ }^{-1}$ is most possibly caused by a large coverage of $\mathrm{H}_{\mathrm{ad}}(>0.6)$. The large coverage of $\mathrm{H}$ is also rendered by theoretical calculation that zero $\Delta G_{\mathrm{H}}{ }^{\circ}$ could be achieved at a certain coverage of $\mathrm{H}$ on $\mathrm{P}$ sites from 2/4 ML to 3/4 ML. In comparison with metal Mo, we can conclude that a simple phosphorization could completely change the reaction route. By extrapolating the Tafel plot to an overpotential of $0 \mathrm{~V}$, the exchange current density can be extracted (Table 1 ). It is found that MoP exhibits a value of $3.4 \times 10^{-2} \mathrm{~mA} \mathrm{~cm}^{-2}, \sim 70$ times higher than that of metal Mo $\left(4.9 \times 10^{-4} \mathrm{~mA} \mathrm{~cm}^{-2}\right)$ and also outperforms $\mathrm{MoS}_{2}$ that has been reported as a promising catalyst for HER. ${ }^{13,20}$ As a non-noble catalyst in bulk form, MoP has achieved comparably competitive performance to $\mathrm{Ni}_{2} \mathrm{P},{ }^{27}$ and is
Table 1 Tafel slope $b$ and exchange current density $j_{0}$

\begin{tabular}{lcl}
\hline & $b\left(\mathrm{mV} \mathrm{dec}^{-1}\right)$ & $j_{\mathrm{o}}\left(\mathrm{mA} \mathrm{cm}^{-2}\right)$ \\
\hline Mo & 92 & $4.9 \times 10^{-4}$ \\
$\mathrm{Mo}_{3} \mathrm{P}$ & 147 & - \\
$\mathrm{MoP}$ in $0.5 \mathrm{M} \mathrm{H}_{2} \mathrm{SO}_{4}$ & 54 & $3.4 \times 10^{-2}$ \\
$\mathrm{MoP}$ in $1 \mathrm{M} \mathrm{KOH}$ & 48 & $4.6 \times 10^{-2}$ \\
$\mathrm{Pt} / \mathrm{C}$ & 33 & 0.63
\end{tabular}

even superior to $\mathrm{MoB}, \mathrm{Mo}_{2} \mathrm{C},{ }^{23} \mathrm{Mo}_{2} \mathrm{C} / \mathrm{CNT}^{25}$ at a similar loading for HER in acidic medium.

Few catalysts can exhibit good activity and stability under both acidic and alkaline conditions. e.g. $\mathrm{Ni}_{2} \mathrm{P}$ was found to deteriorate rapidly in alkaline medium. ${ }^{27}$ Herein we further examined the activity of MoP in both acidic and alkaline conditions using an electrochemical impedance spectroscopy (EIS) technique. Bode plots of MoP (Fig. S7†) suggest a one-timeconstant process in both acidic and alkaline media and Nyquist plots show a depressed arc intercepted by the $x$-axis in Fig. $4 \mathrm{c}$ and $\mathrm{d}$. The high-frequency intersection with the $x$-axis represents ohmic resistance, mainly arising from the electrolyte and all contact resistances. To decouple the ohmic resistance from the polarization resistance, we applied a model of ohmic resistance $\left(R_{\mathrm{S}}\right)$ in series with a module, where the polarization resistance $\left(R_{\mathrm{ct}}\right)$ is in parallel with a constant phase element (CPE). As summarized in Table $\mathrm{S} 4, \dagger \mathrm{MoP}$ performs slightly better in an alkaline medium than in an acidic medium, as indicated by a smaller $R_{\mathrm{ct}}$ at an overpotential of $50 \mathrm{mV}$ and $100 \mathrm{mV}$. The polarization curve in Fig. 5a agrees with the EIS results. At low current density, MoP in an alkaline medium outperforms that in an acidic medium, and exhibits a nearly overlapping polarization curve in the high-current region, suggesting a good performance in alkaline medium.

Durability is a key factor in evaluating catalyst performance. Long-term stability is investigated both in $1 \mathrm{M} \mathrm{KOH}$ and $0.5 \mathrm{M} \mathrm{H}_{2} \mathrm{SO}_{4}$. Unfortunately, slow corrosion happens in 1 $\mathrm{M} \mathrm{KOH}$ as shown in the amperometric plot (Fig. 5b) and the performance degrades to $c a$. $60 \%$ of its initial value in $40 \mathrm{~h}$ (Fig. 5a). However, chronoamperometric electrolysis provides evidence of the excellent stability of MoP in $0.5 \mathrm{M} \mathrm{H}_{2} \mathrm{SO}_{4}$ at an applied potential of $0.14 \mathrm{mV} v s$. RHE in Fig. $5 \mathrm{~b}$, and no performance degradation was observed after electrolysis for more than $40 \mathrm{~h}$. The polarization curve after electrolysis shows the slightly enhanced performance in the region of low current density, which could be ascribed to surface activation in cathodic polarization. In contrast, $\mathrm{Mo}$ and $\mathrm{Mo}_{3} \mathrm{P}$ suffer from severe performance degradation in both acidic and alkaline media. Inspection of the morphology after the stability test shows no difference compared to the one before the test (Fig. S8†) in a panoramic view.

Mo is widely known as a chemically unstable metal in acidic media, and with insufficient phosphorization, the degradation of current density was observed even in the second scan of the polarization curve of $\mathrm{Mo}_{3} \mathrm{P}$ (not shown), implying that corrosion occurs. Stability is commonly correlated with structure. As 

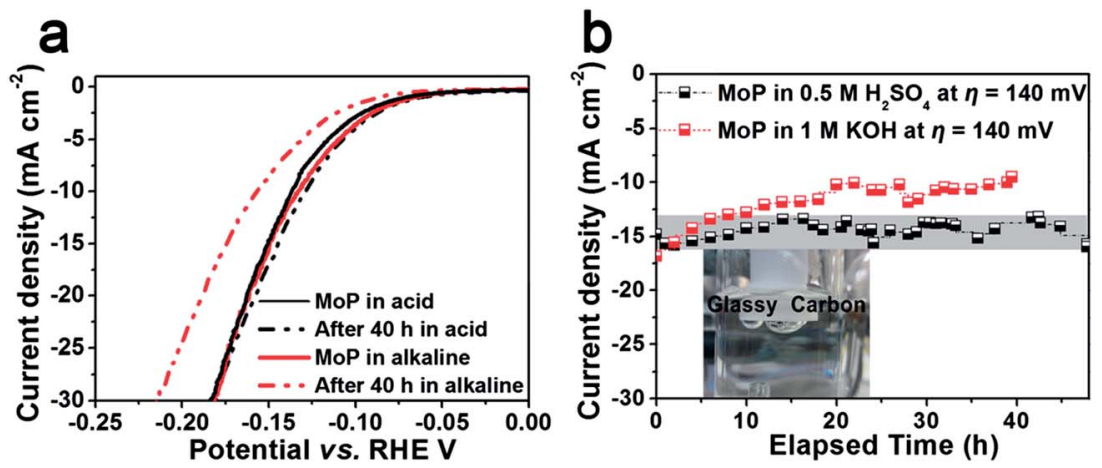

Fig. 5 (a) The polarization curve before and after durability tests, scan rate: $2 \mathrm{mV} \mathrm{s}^{-1}$; (b) chronoamperometric electrolysis.

shown in Scheme 1, the Mo atom is 6-coordinated by $\mathrm{P}$ atom in $\mathrm{MoP}$ in contrast to 2 or $4 \mathrm{P}$-atoms bonded to $\mathrm{Mo}$ in $\mathrm{Mo}_{3} \mathrm{P}$, which could probably be related to the stability in acidic or alkaline media, and suggests the significance of phosphorization.

\section{Conclusions}

Distinct performance was observed from a traditional metal Mo phosphide compound. Our results show catalysts having a higher degree of phosphorization as in MoP results in better performance compared to a lower degree of phosphorization or lack thereof as in $\mathrm{Mo}_{3} \mathrm{P}$ and Mo respectively. Performance in this case is determined based on polarization, Tafel analysis in the low-current region, and stability tests by chronoamperometric electrolysis.

DFT calculations verified that the active site should be ascribed to $\mathrm{P}$ atoms, which achieve a nearly zero Gibbs free energy. The results show that $\mathrm{P}$ atoms have a role analogous to the $\mathrm{S}$ atoms in $\mathrm{MoS}_{2}$, which are responsible for creating a high numbers of edges for HER. ${ }^{19}$ In summary, we have demonstrated that facile phosphorization can transform metal Mo, a poor catalyst, into MoP, an active and stable catalyst for HER. Similar to organic-ligand-functionalized metals, e.g. nickel complexes, ${ }^{7}$ molybdenum-oxo, ${ }^{5}$ this strategy could be easily extended to other earth-abundant and inexpensive metals (e.g. $\mathrm{Ni}, \mathrm{Fe}$, etc.).

\section{Acknowledgements}

Financial support from the academic research fund AcRF tier 1 (M4011020 RG8/12) Ministry of Education, Singapore and competitive research program (2009 NRF-CRP 001-032), National Research Foundation, Singapore. The support by the Singapore National Research Foundation under its Campus for Research Excellence And Technological Enterprise (CREATE) programme is also acknowledged.

\section{References}

1 J. A. Turner, Science, 2004, 305, 972-974.

2 R. Cammack, Nature, 1999, 397, 214-215.
3 A. Volbeda and J. C. Fontecilla-Camps, Dalton Trans., 2003, 4030-4038.

4 S. Shima, O. Pilak, S. Vogt, M. Schick, M. S. Stagni, W. MeyerKlaucke, E. Warkentin, R. K. Thauer and U. Ermler, Science, 2008, 321, 572-575.

5 H. I. Karunadasa, C. J. Chang and J. R. Long, Nature, 2010, 464, 1329-1333.

6 A. Le Goff, V. Artero, B. Jousselme, P. D. Tran, N. Guillet, R. Métayé, A. Fihri, S. Palacin and M. Fontecave, Science, 2009, 326, 1384-1387.

7 P. D. Tran, A. Le Goff, J. Heidkamp, B. Jousselme, N. Guillet, S. Palacin, H. Dau, M. Fontecave and V. Artero, Angew. Chem., Int. Ed., 2011, 50, 1371-1374.

8 D. Merki and X. Hu, Energy Environ. Sci., 2011, 4, 38783888.

9 I. Hatay, P. Y. Ge, H. Vrubel, X. Hu and H. H. Girault, Energy Environ. Sci., 2011, 4, 4246-4251.

10 H. Vrubel, D. Merki and X. Hu, Energy Environ. Sci., 2012, 5, 6136-6144.

11 J. Xie, H. Zhang, S. Li, R. Wang, X. Sun, M. Zhou, J. Zhou, X. W. Lou and Y. Xie, Adv. Mater., 2013, 25, 5807-5813.

12 Z. Chen, D. Cummins, B. N. Reinecke, E. Clark, M. K. Sunkara and T. F. Jaramillo, Nano Lett., 2011, 11, 4168-4175.

13 J. Kibsgaard, Z. Chen, B. N. Reinecke and T. F. Jaramillo, Nat. Mater., 2012, 11, 963-969.

14 M. A. Lukowski, A. S. Daniel, F. Meng, A. Forticaux, L. Li and S. Jin, J. Am. Chem. Soc., 2013, 135, 10274-10277.

15 D. Merki, S. Fierro, H. Vrubel and X. Hu, Chem. Sci., 2011, 2, 1262-1267.

16 J. D. Benck, Z. Chen, L. Y. Kuritzky, A. J. Forman and T. F. Jaramillo, ACS Catal., 2012, 2, 1916-1923.

17 R. R. Chianelli, M. H. Siadati, M. P. De la Rosa, G. Berhault, J. P. Wilcoxon, R. Bearden and B. L. Abrams, Catal. Rev. Sci. Eng., 2006, 48, 1-41.

18 B. Hinnemann, P. G. Moses, J. Bonde, K. P. Jørgensen, J. H. Nielsen, S. Horch, I. Chorkendorff and J. K. Nørskov, J. Am. Chem. Soc., 2005, 127, 5308-5309.

19 T. F. Jaramillo, K. P. Jørgensen, J. Bonde, J. H. Nielsen, S. Horch and I. Chorkendorff, Science, 2007, 317, 100-102.

20 D. Kong, H. Wang, J. J. Cha, M. Pasta, K. J. Koski, J. Yao and Y. Cui, Nano Lett., 2013, 13, 1341-1347. 
21 H. Wang, D. Kong, P. Johanes, J. J. Cha, G. Zheng, K. Yan, N. Liu and Y. Cui, Nano Lett., 2013, 13, 3426-3433.

22 W.-F. Chen, K. Sasaki, C. Ma, A. I. Frenkel, N. Marinkovic, J. T. Muckerman, Y. Zhu and R. R. Adzic, Angew. Chem., Int. Ed., 2012, 51, 6131-6135.

23 H. Vrubel and X. Hu, Angew. Chem., Int. Ed., 2012, 51, 1270312706.

24 W.-F. Chen, S. Iyer, S. Iyer, K. Sasaki, C.-H. Wang, Y. Zhu, J. T. Muckerman and E. Fujita, Energy Environ. Sci., 2013, 6, 1818-1826.

25 W.-f. Chen, C. H. Wang, K. Sasaki, N. Marinkovic, W. Xu, J. T. Muckerman, Y. Zhu and R. R. Adzic, Energy Environ. Sci., 2013, 6, 943-951.

26 L. Liao, S. Wang, J. Xiao, X. Bian, Y. Zhang, M. D. Scanlon, X. Hu, Y. Tang, B. Liu and H. H. Girault, Energy Environ. Sci., 2014, 7, 387-392.

27 E. J. Popczun, J. R. McKone, C. G. Read, A. J. Biacchi, A. M. Wiltrout, N. S. Lewis and R. E. Schaak, J. Am. Chem. Soc., 2013, 135, 9267-9270.

28 L. Feng, H. Vrubel, M. Bensimon and X. Hu, Phys. Chem. Chem. Phys., 2014, 16, 5917-5921.

29 D. Kong, J. J. Cha, H. Wang, H. R. Lee and Y. Cui, Energy Environ. Sci., 2013, 6, 3553-3558.
30 J. K. Nørskov, T. Bligaard, A. Logadottir, J. R. Kitchin, J. G. Chen, S. Pandelov and U. Stimming, J. Electrochem. Soc., 2005, 152, J23-J26.

31 W. Schmickler and S. Trasatti, J. Electrochem. Soc., 2006, 153, L31-L32.

32 J. K. Nørskov, T. Bligaard, A. Logadottir, J. R. Kitchin, J. G. Chen, S. Pandelov and U. Stimming, J. Electrochem. Soc., 2006, 153, L33.

33 P. Liu and J. A. Rodriguez, J. Am. Chem. Soc., 2005, 127, 14871-14878.

34 X. Zhao, M. Cao, B. Liu, Y. Tian and C. Hu, J. Mater. Chem., 2012, 22, 13334-13340.

35 O. G. Marin Flores and S. Ha, Appl. Catal., A, 2009, 352, 124132.

36 J. Bai, X. Li, A. Wang, R. Prins and Y. Wang, J. Catal., 2012, 287, 161-169.

37 D. C. Phillips, S. J. Sawhill, R. Self and M. E. Bussell, J. Catal., 2002, 207, 266-273.

38 Y. Li, H. Wang, L. Xie, Y. Liang, G. Hong and H. Dai, J. Am. Chem. Soc., 2011, 133, 7296-7299.

39 A. B. Laursen, S. Kegnaes, S. Dahl and I. Chorkendorff, Energy Environ. Sci., 2012, 5, 5577-5591.

40 J. G. N. Thomas, Trans. Faraday Soc., 1961, 57, 1603-1611. 